\title{
Évaluation du BactoScan 8000 pour la numération automatique et rapide de la flore microbienne du lait cru
}

\author{
A Dasen, RM Olid, C Piton-Malleret, R Grappin \\ INRA, Station de recherches en technologie et analyses laitières, \\ BP 89, 39801 Poligny Cedex, France
}

(Reçu le 21 mai 1991; accepté le 19 août 1991)

\begin{abstract}
Résumé - Pour évaluer les caractéristiques analytiques de la nouvelle version 8000 du BactoScan (Foss Electric), 578 échantillons de lait cru prélevés à la ferme ont été analysés en double par le BactoScan 8000 , et soumis à la numération de la flore aérobie mésophile, conformément aux recommandations de la norme FIL 100A. Le pourcentage de contamination d'un échantillon sur l'autre a été estimé en analysant de l'eau stérile et un échantillon de lait selon la séquence : lait-eau-lait-eau-etc; sur 33 échantillons, il est en moyenne de $0,16 \%$. Quel que soit le niveau de contamination du lait, la relation entre les résultats obtenus par le BactoScan (BS en log impulsions $/ \mathrm{ml}$ ) et la technique de référence (SPC en log UFC/ml) s'ajuste mieux à un modèle de régression quadratique qu'à un modèle de régression linéaire; l'équation de la courbe de calibration, établie sur les 563 échantillons contenant moins de $510^{5} \mathrm{UFC} / \mathrm{ml}$, est la suivante : SPC $=-0,1619$ $\mathrm{BS}^{2}+2,3921$ BS $-3,2313$, avec un écart type résiduel de 0,2765 log UFC/ml. Le BactoScan surestime en moyenne de $115 \%$ les nombres d'UFC/ml et la limite de détermination de l'appareil a été fixée au-dessous de $10^{4} \mathrm{UFC} / \mathrm{ml}$. L'écart type de répétabilité du BactoScan $s_{\mathrm{r}}{ }^{*}$ exprimé en log UFC/ml est en moyenne de 0,07 , ce qui correspond à un écart type relatif géométrique exprimé en $\%$ d'UFC/ml (ou GRSD) de 17,5\%; il varie en fonction du niveau de contamination du lait et au-dessous de $10^{4} \mathrm{UFC} / \mathrm{ml}$, le GRSD dépasse $25 \%$. Pour tester la stabilité du BactoScan, une suspension de $S$ thermophilus et 3 échantillons de lait cru additionnés d'un conservateur ont été analysés à 3 ou 4 reprises au cours de la journée, et ce, pendant 2 à 3 jours successifs. Le GRSD de reproductibilité de l'appareil sur une courte période (en \% UFC/ml) est généralement inférieur à $20 \%$.
\end{abstract}

BactoScan / lait cru / numération bactérienne / justesse / répétabilité / stabilité / limite de détermination

Summary - Assessment of BactoScan 8000 for the rapid and automatic enumeration of total flora in raw milk. A series of 578 farm raw milk samples has been analysed in duplicate by the BactoScan 8000 and by the reference standard plate count method, according to the IDF standard $100 \mathrm{~A}$. The carry-over effect of BactoScan averages $0.16 \%$. Whatever the level of milk contamination, the relationship between results obtained by the BactoScan (BS in log impulses $/ \mathrm{ml}$ ) and the reference method (SPC in log CFU/ml) is better adjusted to a quadratic regression model than to a linear regression model; for the 563 samples containing less than $510^{5} \mathrm{CFU} / \mathrm{ml}$, the equation of the calibration curve is the following: $S P C=-0.1619 B S^{2}+2.3921 B S-3.2313$, with a residual standard deviation of $0.2765 \mathrm{log}$ CFU/mI. BactoScan overestimates the numbers of CFU, from $115 \%$ on average, and the determination limit is very low: under $10^{4} \mathrm{CFU} / \mathrm{ml}$. The repeatability standard deviation of BactoScan $\mathrm{s}_{r}{ }^{*}$, expressed in log CFU/ml, averages 0.07 , corresponding to a geometric relative 
standard deviation in \% CFU/ml (or GRSD) of 17.5\%; it becomes proportionally higher when the level of the milk contamination decreases and the GRSD is over $25 \%$ for samples under $10^{4} \mathrm{CFU} / \mathrm{ml}$. To test the stability of BactoScan 8000 over a short period, a suspension of S thermophilus and 3 preserved raw milk samples have been analysed 3 or 4 times per day and during 2 or 3 consecutive days. The reproducibility geometric relative standard deviation of BactoScan over a short period (in \% CFU/ml) is generally smaller than $20 \%$.

\section{BactoScan / raw milk / bacterial count / accuracy / repeatability / determination limit / stability}

\section{INTRODUCTION}

Depuis 1986, le BactoScan peut être employé en France comme méthode officielle pour le paiement du lait au producteur, en remplacement de la méthode simplifiée de dénombrement des germes totaux décrite par Thompson et al (1960). Le BactoScan présente de nombreux avantages, en particulier sa rapidité de réponse, son automatisation complète et ses performances analytiques. Ainsi, d'après Grappin et al (1985), sa précision d'estimation est de \pm $0,580 \log U F C / m l$ et son écart type relatif géométrique de répétabilité en moyenne de $5,9 \%$.

Malgré ces avantages, l'implantation de cette technique dans les laboratoires interprofessionnels laitiers français est restée limitée. Outre des considérations économiques, quelques raisons techniques peuvent en être à l'origine, comme sa limite de détermination assez élevée (entre 30 et $50000 \mathrm{UFC} / \mathrm{ml}$ selon les auteurs) et sa cadence d'analyse assez faible (environ 60 échantillons/h).

Récemment, la société Foss Electric a développé une nouvelle version du BactoScan, appelée BactoScan 8000 , dans le but d'améliorer la cadence de passage des échantillons et d'abaisser sa limite de détermination au-dessous de 30000 UFC/ $\mathrm{ml}$. L'objet de cet article est de présenter les résultats d'une étude destinée à évaluer les nouvelles caractéristiques analytiques de cet appareil.

\section{MATÉRIEL ET MÉTHODES}

\section{Origine des échantillons}

578 échantillons de lait cru ont été prélevés et conservés à $0^{\circ} \mathrm{C}$ au plus $24 \mathrm{~h}$ après leur prélèvement. Parmi ces échantillons, 416 provenaient du mélange du lait d'une seule traite, et 162 du mélange habituel du lait de 2 traites.

\section{Numérations microbiennes}

Avant ensemencement, chaque échantillon de lait a subi, dans un flacon de $60 \mathrm{ml}$ rempli au demi, une agitation standard consistant en 25 mouvements aller-retour d'une amplitude de 25 à $30 \mathrm{~cm}$, effectués en 7 à $10 \mathrm{~s}$ (Anonyme, 1978).

Les échantillons ainsi agités ont ensuite été soumis au dénombrement de la flore aérobie mésophile sur milieu Plate Count Agar (Difco), après 3 jours d'incúbation à $30^{\circ} \mathrm{C}$, conformément aux recommandations de la norme FIL 100A (Anonyme, 1987). Chaque échantillon a été dilué au $1 / 100$ et $1 / 1000$ dans des tubes contenant $9 \mathrm{ml}$ de solution de Ringer diluée au $1 / 4$ et les tubes ont été agités pendant $10 \mathrm{~s}$ à l'aide d'un Vortex. Deux boîtes de Petri ont été ensemencées pour chaque dilution.

Après incubation, seules les boîtes ayant entre 10 et 500 colonies ont été retenues pour le comptage. II en résulte que les 15 échantillons contenant plus de $510^{5} \mathrm{CFU} / \mathrm{ml}$ ont été éliminés pour le calcul de la justesse et du seuil de détermination de l'appareil. Les résultats sont exprimés en unités formant colonies par millilitre de lait (UFC/ml). 


\section{BactoScan 8000}

Le principe du BactoScan 8000 reste identique à celui de l'ancienne version, c'est-à-dire un dénombrement automatique des bactéries du lait cru par microscopie épifluorescente après traitement chimique des échantillons. Son fonctionnement a déjà été décrit par Grappin et al (1985).

L'ancienne version a cependant subi quelques modifications. Tout d'abord, pour abaisser le seuil de détermination, la vitesse du disque recevant l'échantillon de lait a été modifiée et l'équipement informatique, dont est maintenant pourvu le BactoScan, permet de régler automatiquement la valeur du discriminateur et d'assurer une meilleure protection contre les bruits de fond. D'autre part, la diminution du temps d'incubation a permis d'écourter le temps de réponse de 7 à 5 min et d'augmenter ainsi la cadence de l'appareil qui est évaluée à 80 échantillons par heure. Enfin, la simplification des circuits et la réduction du nombre et du volume des réactifs rendent l'appareil d'une approche plus facile pour les utilisateurs.

Dans le cadre de cette étude, un échantillon d'eau saline était intercalé entre chaque échantillon de lait analysé afin d'éliminer la contamination éventuelle d'un échantillon par celui qui le précède. Les échantillons étaient préchauffés pendant 10 min à $40^{\circ} \mathrm{C}$ avant passage sur l'appareil et toutes les déterminations étaient faites en double exemplaire.

Les résultats BactoScan sont exprimés en nombre d'impulsions par millilitre de lait (lecture directe $\times 10^{3}$ ).

\section{Stabilité de l'appareil}

Pour déterminer la stabilité du BactoScan 8000 au cours du temps, 3 types d'échantillons ont été utilisés :

- une suspension microbienne de $S$ thermophilus dans une solution de Ringer diluée au 1/4 et conservée pendant 10 jours à $4{ }^{\circ} \mathrm{C}$;

- trois échantillons de lait cru de grand mélange dont la croissance microbienne a été inhibée par addition d'un conservateur, de la composition suivante (Pettipher et Rodrigues, 1982) :

acide borique : $5 \mathrm{~g}$, glycérol : $1 \mathrm{ml}$, sorbate de potassium : $0,075 \mathrm{~g}$, nystatine : 24000 unités, eau distillée : $100 \mathrm{ml}$.

Ce conservateur est ajouté à l'échantillon à la concentration de $20 \mathrm{ml}$ pour $100 \mathrm{ml}$ de lait. Ces échantillons ont été conservés à $4{ }^{\circ} \mathrm{C}$ pendant 2 à 3 jours;

- six échantillons de lait cru de grand mélange sans addition de conservateur et conservés pendant $24 \mathrm{~h}$ dans de la glace fondante.

Tous ces échantillons ont été analysés par le BactoScan 8000 à 3 ou 4 reprises au cours de la journée et à chaque fois, 3 à 4 répétitions ont été effectuées. Dans le cas des échantillons de lait additionnés de conservateur, ce schéma s'est poursuivi pendant 2 à 3 jours successifs; dans le cas de la suspension de $S$ thermophilus, pendant 6 jours répartis sur 2 semaines consécutives.

\section{Calculs statistiques}

Conformément aux recommandations de la norme FIL 128 (Anonyme, 1985), l'évaluation du BactoScan 8000 a comporté d'une part, la vérification d'une caractéristique instrumentale de l'appareil, le degré de contamination d'un échantillon sur l'autre (ou carry-over effect) et d'autre part, la détermination de 3 caractéristiques analytiques importantes : seuil de détermination, justesse et répétabilité.

Avant les calculs statistiques, les nombres d'UFC/ml et d'impulsions/ml ont subi une transformation logarithmique de base 10 pour tenter de normaliser les distributions. D'autre part, les courbes d'ajustement ont été établies à partir des logarithmes décimaux des moyennes arithmétiques des 2 déterminations faites sur le BactoScan.

\section{Seuil de détermination}

Le seuil de détermination d'une technique expérimentale correspond à la limite au-dessus de laquelle cette technique permet d'évaluer la valeur vraie de la grandeur à mesurer avec une incertitude acceptable. II n'existe pas actuellement de norme pour le calcul de ce seuil. Nous nous sommes donc inspirés d'une méthode proposée par Reichmut et al dans un document de travail du groupe FIL E29 (Anonyme, 1990). Ces au- 
teurs proposent d'évaluer ce seuil par itérations successives, en utilisant un modèle de régression quadratique progressive. Ce seuil correspond alors au niveau à partir duquel le modèle devient et reste linéaire, c'est-à-dire le niveau à partir duquel le coefficient de régression de la variable $(\log \mathrm{UFC} / \mathrm{ml})^{2}$ devient non significativement différent de zéro.

Nous avons donc procédé de la manière suivante. Les logarithmes décimaux des nombres d'impulsions/ml sont placés en variable expliquée, de façon à pouvoir fixer le seuil de détermination en UFC/ml. À chaque itération, 10 observations correspondant aux plus faibles niveaux de concentration microbienne sont supprimées et une régression quadratique progressive est effectuée sur les observations restantes, jusqu'à ce que le modèle devienne et reste linéaire.

La limite de détermination est exprimée en $\log U \mathrm{FC} / \mathrm{ml}$ et son équivalence en log impulsions $/ \mathrm{ml}$ peut être calculée à partir de l'équation de calibrage.

\section{Justesse}

La justesse représente l'étroitesse de l'accord entre la valeur vraie de la grandeur à mesurer (par convention, le nombre d'UFC/ml obtenu selon les techniques classiques de dénombrement) et le résultat moyen qui serait obtenu en appliquant la technique expérimentale un grand nombre de fois (Anonyme, 1985).

Conformément à la norme FIL, les logarithmes décimaux des nombres d'UFC/ml sont considérés comme variable dépendante et les logarithmes décimaux des nombres d'impulsions $/ \mathrm{ml}$ comme variable indépendante, pour donner une estimation de l'écart type résiduel dans l'unité de la technique de référence.

La précision d'estimation de la technique expérimentale est donnée par les limites de l'intervalle de confiance à l'intérieur duquel se situe, avec une probabilité de $95 \%$, la vraie valeur donnée par la méthode de référence. Si on appelle y le nombre d'UFC/ml calculé à partir de l'équation de calibrage, ces limites sont, en valeur logarithmique, $\log y \pm 1,96 \times s_{y, x}$ où $s_{y, x}$ est l'écart type résiduel des écarts à la droite de régression. En données non transformées, ces limites sont y $/ 10^{1,96 * s_{y x}}$ pour la limite inférieure et $y 10^{1.96{ }^{*} s_{y, x}}$ pour la limite superieure.

\section{Répétabilité}

Pour pouvoir comparer valablement la répétabilité du BactoScan 8000 avec celle d'autres techniques analytiques, notamment la technique de référence, l'écart type de répétabilité a été exprimé en log UFC/ml. Pour cela, les résultats des 2 déterminations effectuées par le BactoScan sur chaque échantillon de numéro i ont été convertis en $\log$ UFC/ml par l'intermédiaire de l'équation de calibrage de l'appareil.

L'écart type de répétabilité du BactoScan $8000\left(s_{r}{ }^{*}\right)$ a été établi à partir de la formule suivante:

$$
s_{\mathrm{r}}{ }^{*}=\left(\Sigma w_{\mathrm{i}}^{2} / 2 n\right)^{1 / 2}
$$

où $w_{i}$ est la valeur absolue de la différence entre doubles pour l'échantillon i (en log UFC/ $\mathrm{ml}$ ) et $n$ le nombre d'échantillons analysés (Anonyme, 1985); l'astérisque signifie que l'écart type est exprimé dans l'unité de la technique de référence.

L'écart type relatif géométrique de répétabilité GRSD en données non transformées est alors estimé à partir de la formule suivante :

$$
\text { GRSD (en UFC/ml) }=\left(10^{S_{r *}}-1\right) \times 100 \text {. }
$$

Ce paramètre exprime la variabilité des résultats au-dessus de la moyenne géométrique (Piton et Grappin, 1991).

\section{Reproductibilité de l'appareil au cours du temps}

Comme précédemment, les nombres d'impulsions $/ \mathrm{ml}$ obtenus sur les échantillons destinés à tester la stabilité de l'appareil au cours du temps ont été convertis en log UFC/ml à l'aide de l'équation de calibrage. Les résultats obtenus sur chaque échantillon ont ensuite été soumis à une analyse de variance à modèle croisé hiérarchisé; les facteurs pris en compte dans le modèle sont les suivants : jour, heure d'analyse et répétition. Les résultats de cette analyse permettent d'estimer la variance de répétabilité de l'appareil $\left(s_{\mathrm{r}}{ }^{* 2}\right)$, la variance entre heures d'analyse dans la même journée $\left(s_{H}{ }^{* 2}\right)$ et la variance entre jours d'analyse $\left(s_{j}{ }^{* 2}\right)$ (Dagnelie, 1970).

A partir de ces variances estimées, il est possible de calculer : 
- la variance de répétabilité de l'appareil, $s_{\mathrm{r}}$ *2;

- la variance de reproductibilité sur une courte période ou reproductibilité intra-jour, grâce à la formule: $s_{\mathrm{R} 1}{ }^{\star 2}=s_{\mathrm{r}}{ }^{\star 2}+s_{\mathrm{H}}{ }^{* 2}$;

- la variance de reproductibilité sur une longue période (2 à 6 jours) ou reproductiblité entrejours, grâce à la formule: $s_{\mathrm{R} 2}{ }^{\star 2}=s_{\mathrm{r}}{ }^{\star 2}+s_{\mathrm{H}}{ }^{\star 2}+$ $s_{J}^{* 2}$.

\section{RÉSULTATS}

\section{Contamination d'un échantillon par l'autre}

Cette caractéristique instrumentale a été estimée dans une étude préliminaire, en analysant de l'eau stérile et un lait chargé en micro-organismes selon la séquence suivante : lait - eau - lait - eau - etc. Sur 33 échantillons de lait analysés suivant le schéma précédent, le taux de contamination d'un échantillon par l'échantillon qui le précède, est en moyenne de $0,16 \%$

\section{Nature de la relation référence-BactoScan}

La figure 1 présente le diagramme de dispersion des résultats obtenus avec le BactoScan 8000 et la méthode de référence. Les logarithmes décimaux des nombres d'impulsions/ml sont placés comme variable dépendante et les logarithmes décimaux des nombres d'UFC/ml comme variable indépendante, afin de permettre de fixer la limite de détermination.

Le nuage de points ainsi construit ne permet pas a priori de juger du caractère de la relation entre les 2 méthodes. Deux modèles de régression ont donc été appliqués à l'ensemble du nuage : un modèle

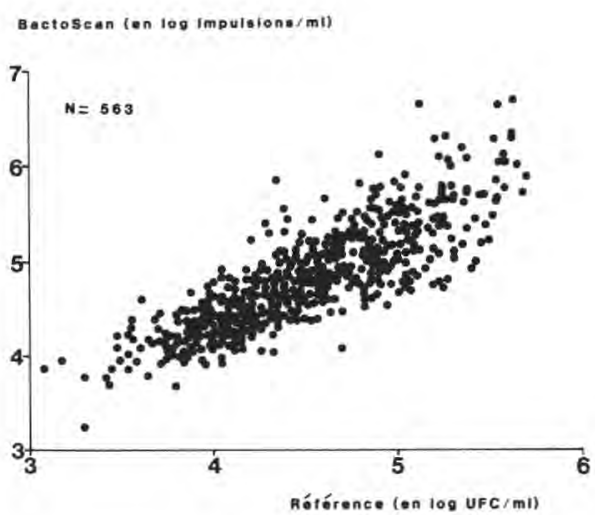

Fig 1. Diagramme de dispersion des résultats obtenus avec la méthode de référence et le BactoScan 8000 .

Scatter diagram of results obtained with the reference method and the BactoScan 8000 .

de régression quadratique progressive et un modèle de régression linéaire. Pour la régression linéaire, l'équation obtenue est la suivante $: \log (B S)=0,8864 \log (R E F)+$ 0,8447 , avec un écart type résiduel $\mathrm{s}_{\mathrm{y}, \mathrm{x}}$ de $0,3025 \mathrm{log}$ impulsions/ml. Dans le cas de la régression quadratique, l'équation obtenue est :

$\log (B S)=0,068(\log (R E F))^{2}+0,27 \log$ $(\mathrm{REF})+2,21$, avec un écart type résiduel $\mathrm{s}_{\mathrm{y}, \mathrm{x}}$ de $0,3020 \log$ impulsions $/ \mathrm{ml}$.

On ne peut rejeter aucun des 2 modèles sur la base des écarts types résiduels car ils ne sont pas significativement différents. Cependant, avec le modèle de régression quadratique progressive, la contribution de la variable $(\log (\mathrm{REF}))^{2}$ se révèle hautement significative $(P<0,001)$. Pour cette raison, nous avons donc retenu la régression quadratique pour décrire la relation entre le BactoScan 8000 et la technique de référence. 


\section{Seuil de détermination}

Le tableau I présente les résultats des calculs effectués pour fixer le seuil de détermination, c'est-à-dire l'équation du modèle le plus approprié pour décrire la relation entre le BactoScan et la technique de référence, après suppression des échantillons 10 par 10 . On peut constater que le modèle change alternativement de degré selon le nombre d'observations supprimées. Cela signifie donc que cette technique statistique ne permet pas de fixer de limite de détermination pour le BactoScan 8000. D'ailleurs, la simple observation visuelle du diagramme montre qu'il n'y a pas de limite de détermination clairement visible, l'écart type résiduel apparaissant plus petit aux faibles concentrations.

\section{Justesse}

L'équation de calibrage a été calculée selon un modèle de régression quadratique pour les deux raisons suivantes : d'une part, sur l'ensemble du nuage de points, nous avons montré que ce modèle permet au mieux de décrire la relation entre les deux techniques et d'autre part, nous n'avons pas pu fixer de limite au-delà de laquelle le modèle devient et reste linéaire. Nous avons préféré utiliser ce modèle plutôt qu'un modèle reposant sur 2 ou 3 droites découpées arbitrairement à 1 ou 2 niveaux de concentration. La courbe de calibration du BactoScan 8000 , d'équation $\log (R E F)=c \log (B S)^{2}+b \log (B S)+a, a$ donc été calculée sur les 563 échantillons contenant moins de $510^{5} \mathrm{UFC} / \mathrm{ml}$. Cette équation est la suivante (fig 2) :

Tableau I. Évaluation du seuil de détermination du BactoScan. Calculation of the determination limit of BactoScan.

\begin{tabular}{|c|c|c|c|c|c|c|}
\hline \multirow{2}{*}{$\frac{N^{a}}{10}$} & \multirow{2}{*}{$\begin{array}{c}\text { Niveau }^{b} \\
3,1\end{array}$} & \multirow{2}{*}{$\begin{array}{c}\begin{array}{c}\text { Variable } \\
\text { significative }\end{array} \\
\log (\mathrm{REF})^{2}\end{array}$} & \multicolumn{4}{|c|}{ Équation du modèle retenu } \\
\hline & & & $\log (B S)$ & $=0,097$ & $\log (R E F)^{2}$ & $+2,84$ \\
\hline 20 & 4,1 & $\log (R E F)$ & $\log (B S)$ & $=0,91$ & $\log (\mathrm{REF})$ & $+0,75$ \\
\hline 30 & 5,4 & $\log (R E F)$ & $\log (B S)$ & $=0,91$ & $\log (R E F)$ & $+0,72$ \\
\hline 40 & 6,2 & $\log (R E F)$ & $\log (B S)$ & $=0,91$ & $\log (\mathrm{REF})$ & $+0,73$ \\
\hline 50 & 6,7 & $\log (\mathrm{REF})$ & $\log (B S)$ & $=0,91$ & $\log (\mathrm{REF})$ & $+0,75$ \\
\hline 60 & 7,4 & $\log (R E F)^{2}$ & $\log (B S)$ & $=0,097$ & $\log (R E F)^{2}$ & $+2,84$ \\
\hline 70 & 7,7 & $\log (R E F)$ & $\log (B S)$ & $=0,91$ & $\log (R E F)$ & $+0,72$ \\
\hline 80 & 8,7 & $\log (R E F)^{2}$ & $\log (B S)$ & $=0,097$ & $\log (R E F)^{2}$ & $+2,85$ \\
\hline 90 & 9,3 & $\log (R E F)$ & $\log (B S)$ & $=0,92$ & $\log (R E F)$ & $+0,69$ \\
\hline 100 & 10,0 & $\log (R E F)$ & $\log (B S)$ & $=0,92$ & $\log (R E F)$ & $+0,67$ \\
\hline 110 & 10,9 & $\log (R E F)$ & $\log (B S)$ & $=0,92$ & $\log (\mathrm{REF})$ & $+0,67$ \\
\hline 120 & 10,9 & $\log (R E F)$ & $\log (B S)$ & $=0,92$ & $\log (R E F)$ & $+0,67$ \\
\hline 130 & 12,0 & $\log (R E F)^{2}$ & $\log (B S)$ & $=0,096$ & $\log (R E F)^{2}$ & $+2,87$ \\
\hline
\end{tabular}

a $\mathrm{N}$ : nombre d'observations supprimées; b Valeur minimale de concentration microbienne en UFC/ml $\left(x 10^{3}\right) ; \log$ (REF) : nombre de micro-organismes/ml en log UFC; $\log (\mathrm{BS})$ : résultats obtenus avec le BactoScan en log impulsions $/ \mathrm{ml}$.

${ }^{2} \mathrm{~N}$ : number of samples deleted; ${ }^{b}$ lowest level of micro-organisms in CFU/ml $\left(\times 10^{3}\right) ; \log (\mathrm{REF})$ : number of microorganisms/ml in log CFU; $\log (B S)$ : Bactoscan readings in log impulsions $/ \mathrm{ml}$. 


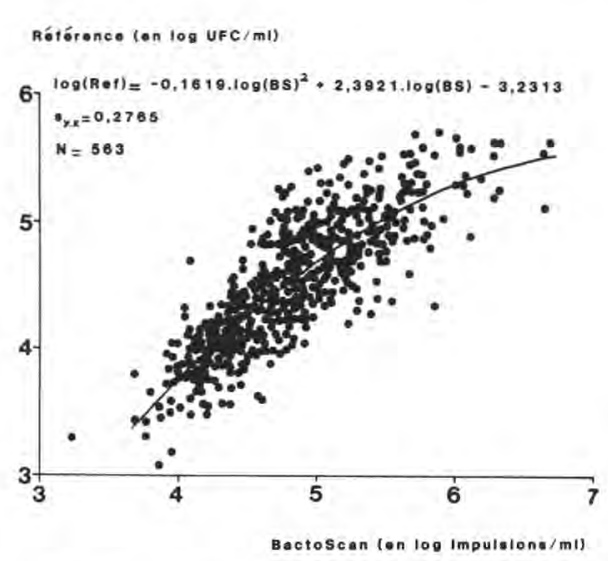

Fig 2. Équation de calibration du BactoScan 8000.

Calibration curve of the BactoScan 8000 .

$\log (\mathrm{REF})=-0,1619(\log (\mathrm{BS}))^{2}+2,3921$ $\log (B S)-3,2313$, avec un écart type résiduel, $s_{y, x}$ de $0,2765 \log$ UFC/ml. Tous les résultats obtenus sur cet appareil doivent donc être convertis en UFC/ml à l'aide de cette équation.

La précision d'estimation du BactoScan 8000 est de $\pm 1,96 \times 0,2765= \pm 0,542 \mathrm{log}$ UFC/ml. Si y est le nombre d'UFC/ml calculé à partir de la droite de calibrage, les limites de l'intervalle de confiance de log y sont, à $P=0,95: \log \mathrm{y}+0,542$ pour la limite supérieure et log $y-0,542$ pour la limite inférieure. En données non transformées, ces limites deviennent : y x 100,542, soit $y \times 3,48$ pour la limite supérieure et $y /$ $10^{0,542}$, soit $y / 3,48$ pour la limite inférieure.

Enfin, sur les 563 échantillons de lait analysés, les moyennes sont respectivement de 4,83 log impulsions/ml pour le BactoScan et de $4,50 \mathrm{log} U \mathrm{FC} / \mathrm{ml}$ pour la référence et elles sont significativement différentes au seuil de $5 \%$. Le BactoScan surestime les nombres d'UFC/ml en moyenne de $115 \%$.

\section{Répétabilité}

Le tableau II présente, pour chaque niveau, les écarts types de répétabilité $\left(s_{r}{ }^{*}\right.$ en log UFC/ml), ainsi que les écarts types relatifs géométriques (GRSD en \% UFC/ $\mathrm{ml})$ du BactoScan 8000 . II présente également les GRSD obtenus par Grappin et al (1985) pour la technique de référence. On observe tout d'abord une nette augmentation de l'écart type de répétabilité du BactoScan quand le niveau de contamination du lait baisse. La répétabilité de cet appareil reste toutefois meilleure que celle de la technique de référence pour les échantillons contenant plus de $25000 \mathrm{UFC} / \mathrm{ml}$. En deçà, les écarts types relatifs géométriques augmentent nettement et ils dépassent $25 \%$ au-dessous de 10000 UFC/ml.

\section{Stabilité de l'appareil au cours du temps}

Le tableau III présente, pour la suspension de $S$ thermophilus et les 3 laits additionnés de conservateur, les écarts types de répétabilité et de reproductibilité obtenus, ainsi que les écarts types relatifs géométriques correspondants. On observe tout d'abord qu'ils sont particulièrement faibles pour la suspension microbienne qui contenait environ $3,610^{6} \mathrm{UFC} / \mathrm{ml}$. À l'inverse, pour les laits, les GRSD de reproductibilité sont nettement plus élevés mais ils restent généralement inférieurs à $20 \%$, traduisant ainsi une grande stabilité de l'appareil au cours du temps. Comme dans le cas de la répétabilité, les valeurs de reproductibilité semblent varier en fonction du niveau de contamination des échantillons. En effet, le 
Tableau II. Répétabilité du BactoScan. Repeatability of the BactoScan.

\begin{tabular}{crcrrr}
\hline $\begin{array}{c}\text { Niveau } \\
\text { en } \begin{array}{c}\text { Moc/ml } \\
\left(\times 10^{3}\right)\end{array}\end{array}$ & $N$ & $\begin{array}{c}\text { Moyenne } \\
\text { BactoScan } \\
\left(\times 10^{3}\right)\end{array}$ & $\begin{array}{c}\mathrm{s}_{r}^{*} \\
\text { BactoScan }\end{array}$ & $\begin{array}{c}\text { GRSD } \\
\text { BactoScan }\end{array}$ & $\begin{array}{r}\text { GRSDa } \\
\text { Référence }\end{array}$ \\
\hline$<10$ & 80 & 7 & 0,132 & 35,5 & nd \\
$10-15$ & 61 & 12 & 0,092 & 23,6 & nd \\
$15-25$ & 88 & 20 & 0,078 & 19,7 & 14,3 \\
$25-50$ & 142 & 35 & 0,051 & 12,4 & 13,9 \\
$50-100$ & 116 & 69 & 0,035 & 8,4 & 11,9 \\
$100-250$ & 74 & 142 & 0,015 & 3,5 & 12,7 \\
$>250$ & 17 & 308 & 0,005 & 1,2 & nd \\
\hline
\end{tabular}

nd : non déterminé; $\mathrm{N}$ : nombre d'échantillons; $s_{\mathrm{r}}^{*}$ : écart type de répétabilité du BactoScan en log UFC/ml; GRSD : écart type relatif géométrique de répétabilité en \% d'UFC/ml; a : GRSD de la technique de référence d'après Grappin et al (1985).

nd: non determined; $N$ : number of samples; $\mathrm{s}_{r}$ : repeatability standard deviation of BactoScan in log CFU/ml; GRSD: geometric relative standard deviation of repeatability in \% CFU/ml; a : GRSD obtained by Grappin et al (1985) for the reference technique.

Tableau III. Stabilité du BactoScan 8000.

Stability of the BactoScan 8000.

\begin{tabular}{|c|c|c|c|c|c|c|c|}
\hline \multirow[b]{2}{*}{ Échantillon } & \multirow[b]{2}{*}{$N$} & \multirow[t]{2}{*}{$\begin{array}{l}\text { Niveau } \\
\text { moyen a }\end{array}$} & \multirow{2}{*}{$\begin{array}{c}\text { Répétabilité } \\
\text { GRSD }\end{array}$} & \multicolumn{2}{|c|}{$\begin{array}{c}\text { Reproductibilité } \\
\text { intra-jour }\end{array}$} & \multicolumn{2}{|c|}{$\begin{array}{c}\text { Reproductibilité } \\
\text { entre-jours }\end{array}$} \\
\hline & & & & $s_{R 1^{*}}$ & $G R S D$ & $s_{R 2^{*}}$ & GRSD \\
\hline $\begin{array}{l}\text { Suspension de } \\
S \text { thermophilus }\end{array}$ & 96 & $3,610^{6}$ & 0,6 & 0,004 & 1,0 & 0,005 & 1,1 \\
\hline \multicolumn{8}{|c|}{$\begin{array}{l}\text { Laits additionnés } \\
\text { de conservateurs }\end{array}$} \\
\hline Lait 1 & 24 & $7,210^{4}$ & 15,3 & 0,086 & 21,9 & 0,086 & 21,9 \\
\hline Lait 2 & 24 & $2,910^{5}$ & 1,6 & 0,007 & 1,6 & 0,035 & 8,4 \\
\hline Lait 3 & 36 & $3,010^{4}$ & 12,5 & 0,053 & 13,0 & 0,053 & 13,0 \\
\hline
\end{tabular}

$\mathrm{N}$ : nombre total d'analyses effectuées sur chacun des échantillons; a : niveau moyen de contamination; GRSD : écart type relatif géométrique de répétabilité ou de reproductibilité en \% UFC/ml; $\mathrm{s}_{\mathrm{R} 1}{ }^{*}$ ou $\mathrm{S}_{\mathrm{R} 2}{ }^{*}$ : écart type de reproductibilité intra-jour ou entre-jours en log UFC/ml.

$N$ : total number of analyses for each sample; a : mean level of contamination; GRSD : geometric relative standard deviation of repeatability or reproductibility in \% CFU/ml; $s_{R 1}{ }^{*}$ or $s_{R 2}{ }^{*}$ : within-day or between-days reproducibility standard deviation in log CFU/ml. 
lait $\mathrm{n}^{\circ} 2$ contenait environ $310^{5} \mathrm{UFC} / \mathrm{ml}$, contre respectivement $310^{4}$ et $7,210^{4}$ UFC/ml pour les 2 autres.

Pour les laits de grand mélange sans addition de conservateur, les écarts types de reproductibilité sur une courte période ( 1 jour) étaient compris entre 0,007 et $0,071 \log U F C / m l$, ce qui correspond à des GRSD variant entre 1,6 et $17,8 \%$.

\section{DISCUSSION}

Cette étude nous a permis d'évaluer les principales caractéristiques analytiques de la nouvelle version du BactoScan 8000 . Tout d'abord, la précision d'estimation de l'appareil est tout-à-fait comparable à celle obtenue par Grappin et al (1985), Nieuwenhof et Hoolwerf (1988) ou Suhren et al (1988). En effet, dans notre étude, elle est de $\pm 0,542 \mathrm{log} U F C / m l$ contre par exemple, $\pm 0,580 \mathrm{log} U F C / \mathrm{ml}$ pour la première version (Grappin et al, 1985).

Conformément à ce qui était attendu, les modifications apportées à l'appareil se sont traduites par un abaissement significatif de sa limite de détermination. Ainsi, à I'inverse de ce qu'ont observé Grappin et al (1985) sur la première version du BactoScan et Nieuwenhof et Hoolwerf (1988) ou Suhren et al (1988) sur la nouvelle version 8000 , nous n'avons pas mis en évidence, pour les échantillons faiblement contaminés, de déviation systématique par rapport au modèle linéaire, qu'on puisse attribuer à des bruits de fond d'origine non bactérienne. En effet, il est apparu qu'à tous les niveaux de pollution du lait, une équation du second degré donnait le meilleur ajustement des réponses Bactoscan en fonction du niveau et que la variance résiduelle était au moins aussi bonne, sinon meilleure, aux faibles niveaux de contamination du lait. Nos résultats ont donc permis de fixer le seuil de dé- termination du BactoScan 8000 à des niveaux faibles de contamination du lait, c'est-à-dire en-dessous de $10^{4} \mathrm{UFC} / \mathrm{ml}$, sans qu'il soit possible de donner une valeur plus précise. Ces conclusions sont en contradiction avec celles données par Nieuwenhof et Hoolwerf (1988) ou Suhren et al (1988) pour le BactoScan 8000; en effet, ces auteurs l'avaient fixé à $310^{4}$ UFC/ml pour les premiers et pour les seconds, entre 4 et $810^{4} \mathrm{UFC} / \mathrm{ml}$ selon le mode de calcul. Des modifications apportées à la nouvelle version 8000 depuis les essais réalisés par ces auteurs, notamment dans le circuit des fluides au niveau de la centrifugeuse, sont à l'origine de cette amélioration du seuil de détermination (Kaereby, communication personnelle).

Par rapport à la majorité des travaux précédents, notre étude apporte des informations plus complètes sur la fidélité du BactoScan, particulièrement pour les très faibles niveaux de contamination microbienne du lait et sur sa stabilité sur une courte et moyenne période. Au-dessus de $510^{4} \mathrm{UFC} / \mathrm{ml}$, les écarts types relatifs géométriques de répétabilité (GRSD) obtenus sont très satisfaisants (moins de 10\%) et assez proches de ceux obtenus sur la première version du BactoScan par Grappin et al (1985). Par contre, dès que le niveau de contamination du lait baisse, nous confirmons la nette augmentation de la répétabilité déjà notée par Grappin et al (1985) et par Suhren et al (1988); celle-ci est probablement liée à la diminution du nombre de particules colorées comptées sur le disque. Cependant, si on considère un GRSD de $25 \%$ comme limite supérieure acceptable, le BactoScan 8000 présente une répétabilité satisfaisante pour tous les échantillons contenant plus de $10^{4}$ UFC/ml.

L'ensemble des résultats permet de conclure que les modifications apportées 
au BactoScan, depuis sa première version, ont conduit à une amélioration de la plupart des performances analytiques. Toutefois, comme l'étude a été réalisée dans de bonnes conditions expérimentales et avec des échantillons de lait âgés au plus de $24 \mathrm{~h}$, des études complémentaires semblent nécessaires avant de pouvoir tirer des conclusions générales.

\section{RÉFÉRENCES}

Anonyme (1978) Standard Methods for the Examination of Dairy Products. American Public Health Association, 14th edn, New York

Anonyme (1985) Milk. Definition and evaluation of the overall accuracy of indirect methods of milk analysis. Application to calibration procedure and quality control in dairy laboratory. Norme provisoire FIL 128

Anonyme (1987) Lait et produits laitiers. Dénombrement des microorganismes. Comptage des colonies à $30^{\circ} \mathrm{C}$. Norme provisoire FIL 100A

Anonyme (1990) Evaluation of routine methods for the quantitative determination of the bacteriological quality of milk. First draft of a working document for the E29 IDF-Group, March 1990
Dagnelie P (1970) Théorie et méthodes statistiques. Vol 2. Les méthodes de l'inférence statistique. Éditions J Duculot, Gembloux

Grappin R, Dasen A, Favennec P (1985) Numération automatique et rapide des bactéries du lait cru à l'aide du BactoScan. Lait 65, 123147

Nieuwenhof FFJ, Hoolwerf JD (1988) Suitability of BactoScan for the estimation of the bacteriological quality of raw milk. Milchwissenschaft 43, 577-586

Pettipher GL, Rodrigues UM (1982) A bacteriostatic mixture for milk samples and its effect on bacteriological, cytological and chemical compositional analysis. J Appl Bacteriol 52, 259-265

Piton C, Grappin R (1991) A model for statistical evaluation of precision parameters of microbiological methods: application to dry rehydratable film methods and IDF reference methods for enumeration of total aerobic mesophilic flora and coliforms in raw milk. $J$ Assoc Off Anal Chem 74, 92-103

Suhren G, Reichmut J, Heeschen W (1988) Zur Messung der Bakteriologischen Beschaffenheit der Rohmilch mit dem BactoScan-Gerät. Erste Erfahrungen und Ergebnisse mit dem BactoScan III-System. Teil I und II. Dtsch Molk Ztg 44, 1436-1446 and 46, 1551-1555

Thompson DI, Donnelly CB, Black LA (1960) A plate loop method for determining viable counts of raw milk. J Milk Food Technol 23, 167-171 